\title{
Higher-Order Contingentism, Part 2: Patterns of Indistinguishability*
}

\author{
Peter Fritz
}

Final Draft

\begin{abstract}
The models of contingency in what propositions, properties and relations there are developed in Part 1 are related to models of contingency in what propositions there are due to Robert Stalnaker. It is shown that some but not all of the classes of models of Part 1 agree with Stalnaker's models concerning the patterns of contingency in what propositions there are they admit. Further structural connections between the two kinds of models are explored.
\end{abstract}

\section{Introduction}

Part 1 (Fritz and Goodman, 2016) explores models for the view that it is a contingent matter what propositions, properties and relations there are, building on work by Fine (1977) and Stalnaker (2012). Stalnaker (2012, Appendix A) focuses in particular on the view that it is a contingent matter what propositions there are, and develops two kinds of models for it. For brevity, I will use the label "propositional contingency" for contingency in what propositions there are, and "higher-order contingency" for contingency in what propositions, properties and relations there are.

This paper investigates connections between the models of higher-order contingency of Part 1 and Stalnaker's models of propositional contingency. Because of their complexity, the required definitions and results of Part 1 will not be repeated here; familiarity with them will be assumed. Stalnaker's models will be introduced in section 2, and familiarity with them is not required. For each of the various classes of models of higher-order contingency investigated in Part 1, the most important question is whether they agree with Stalnaker's models on the patterns of propositional contingency they admit. This issue is investigated in section 3. The results obtained there are mainly positive: For several - but not all - classes, it will be shown that the patterns of propositional contingency they admit are precisely the patterns of propositional contingency Stalnaker's models admit. Some of Stalnaker's models record not only patterns of propositional contingency, but also patterns of a kind of permutability of worlds. These latter patterns can also be obtained from models of higher-order contingency, and are explored in more detail in section 4 . Section 5 concludes, and highlights some consequences of the results obtained here.

\footnotetext{
*Forthcoming in the Journal of Philosophical Logic. The final publication is available at https://link.springer.com/article/10.1007/s10992-017-9432-3.
} 


\section{Equivalence Systems and Permutation Systems}

The models of Stalnaker (2012, Appendix A) are developed in more detail in Fritz (2016); as Stalnaker (2016, p. 725, fn. 15) notes, the latter also corrects some errors of the former. This section summarizes the relevant definitions and results of Fritz (2016); readers familiar with this article may skip this section, and readers looking for a more in-depth discussion of the material presented here may find it useful to consult the article.

Stalnaker develops two kinds of models. Models of the first kind will be called equivalence systems. In such models, every member of a set of worlds is mapped to an equivalence relation on the set of worlds:

Definition 2.1. For every set $W$, an equivalence system on $W$ is a function $\approx$ mapping every $w \in W$ to an equivalence relation $\approx_{w}$ on $W$.

One can think of these systems as representing which pairs of worlds are indistinguishable at a given world. A proposition $p \subseteq W$ is taken to exist at a world $w$ of an equivalence system $\approx$ just in case $p$ does not distinguish between any worlds indistinguishable at $w$, i.e., just in case $v \approx_{w} u$ entails that $v \in p$ iff $u \in p$. Each equivalence system is therefore naturally mapped to a propositional domain function, i.e., a function mapping worlds to sets of propositions; indeed, it is easy to see that this mapping is a one-to-one correspondence between equivalence systems and propositional domain functions mapping each world to a complete atomic field of sets.

Some equivalence systems are implausible models of propositional contingency, as they count some worlds $v$ and $u$ as indistinguishable at $w$, even though $v$ and $u$ can intuitively be distinguished at $w$, e.g., in terms of the indistinguishability relations associated with them. To rule out these cases, a coherence constraint is formulated using some basic algebraic notions. A permutation of a set $W$ is a bijection from $W$ to $W$. An automorphism of an equivalence system $\approx$ on a set $W$ is a permutation $f$ of $W$ such that $v \approx_{w} u$ iff $f(v) \approx_{f(w)} f(u)$, for all $w, v, u \in W$. aut $(\approx)$ is the set of automorphisms of $\approx$, and $\operatorname{aut}(\approx)_{w}$ is the set of automorphisms of $\approx$ mapping $w$ to itself. Both are permutation groups on $S$, i.e., subgroups of the symmetric group $S_{W}$. $\left(S_{W}\right.$ is the set of permutations of $W$; its subgroups are its nonempty subsets which are closed under composition and inverses.) aut $(\approx)_{w}$ is called the stabilizer of aut $(\approx)$ with respect to $w$.

Definition 2.2. An equivalence system $\approx$ on a set $W$ coheres if for all $w, v, u \in$ $W$ such that $v \approx_{w} u$, there is an $f \in \operatorname{aut}(\approx)_{w}$ such that $f(v)=u$ and $f \subseteq \approx_{w}$.

Models of Stalnaker's second kind will be called permutation systems. In such models, every member of a set of worlds is mapped to a permutation group on the set of worlds:

Definition 2.3. For every set $W$, a permutation system on $W$ is a function $F$ mapping every $w \in W$ to a permutation group $F_{w}$ on $W$.

One can think of these systems as representing how the worlds can be permuted in ways indistinguishable at a given world. As in the case of equivalence systems, structurally implausible permutation systems are ruled out by a condition of coherence. Formulating this condition requires a couple of additional basic group-theoretic concepts. For any permutations $f$ and $g$ of a set $W$, let $f . g$ 
be $f g f^{-1}$ (the conjugation of $g$ by $f$ ), and for any set of permutations $G \subseteq S_{W}$, let $f . G$ be $\{f . g: g \in G\}$. Let a permutation $f$ of a set $W$ be an automorphism of a permutation system $F$ on $W$ if $f . F_{w}=F_{f(w)}$ for all $w \in W$. Let aut $(F)$ be the set of automorphisms of $F$ and $\operatorname{aut}(F)_{w}$ the stabilizer of $\operatorname{aut}(F)$ with respect to $w$, i.e., the set of automorphisms of $F$ which map $w$ to itself.

Definition 2.4. A permutation system $F$ on a set $W$ coheres if for all $w \in W$, $F_{w} \subseteq \operatorname{aut}(F)_{w}$.

Permutation systems determine equivalence systems in a straightforward way, since worlds $v$ and $u$ may be counted as indistinguishable at $w$ just in case there is a way of permuting worlds indistinguishably at $w$ which maps $v$ to $u$. Via the equivalence systems they determine, permutation systems thus also model propositional contingency. Indeed, the coherence constraints on the two kinds of systems agree on the patterns of propositional contingency they admit, as it can be shown that an equivalence system is coherent just in case it is determined by a coherent permutation system. However, equivalence systems and permutation systems are not interchangeable, since there are distinct coherent permutation systems which determine the same (coherent) equivalence system. This is not surprising: which pairs of worlds are indistinguishable at a given world is plausibly determined by how the worlds can be permuted overall in ways which are indistinguishable at a given world, but there is no reason to expect the latter to be determined by the former. If we say that equivalence systems record patterns of local indistinguishability, and permutation systems record patterns of global indistinguishability, this observation can be summed up by saying that global indistinguishability goes beyond local indistinguishability.

The following two sections derive these two kinds of patterns of indistinguishability from the models of Part 1, comparing the resulting classes of equivalence and permutation systems to those identified as coherent.

\section{Local Indistinguishability}

Recall that a structure in the sense of Part 1 is a tuple $\mathfrak{S}=\langle W, I, D\rangle$, where $W$ is the set of worlds, $I$ the set of individuals, and $D$ a variable domain function mapping each $w \in W$ and type $t$ to a set $D_{w}^{t}$. For any $w \in W$, $\operatorname{fix}(\mathfrak{S}, w)$ is the set of automorphisms of $\mathfrak{S}$ mapping $w$ and all the elements of the domains (of the different types) of $w$ to themselves; here, an automorphism of a structure is a pair consisting of a permutation of worlds and a permutation of individuals. The elements of $\operatorname{fix}(\mathfrak{S}, w)$ can therefore be understood as recording the ways modal space can be permuted in ways indistinguishable at $w$ in $\mathfrak{S}$. So, similar to the determination of equivalence systems by permutation systems, a structure determines an equivalence system by letting $v$ and $u$ be indistinguishable at $w$ just in case there is an automorphism in $\operatorname{fix}(\mathfrak{S}, w)$ mapping $v$ to $u$ :

Definition 3.1. For any structure $\mathfrak{S}=\langle W, I, D\rangle$, the equivalence system determined by $\mathfrak{S}$ is the function $\varepsilon(\mathfrak{S})$ such that for all $w, v, u \in W$,

$$
v \varepsilon(\mathfrak{S})_{w} u \text { iff there is } a \xi \in \operatorname{fix}(\mathfrak{S}, w) \text { such that } \xi . v=u \text {. }
$$

Equivalence systems derived in this way are always coherent:

Proposition 3.2. For any structure $\mathfrak{S}, \varepsilon(\mathfrak{S})$ is a coherent equivalence system. 
Proof. Let $w \in W$. That $\varepsilon(\mathfrak{S})_{w}$ is an equivalence relation follows from the fact that $\operatorname{fix}(\mathfrak{S}, w)$ is a permutation group, as noted in Part 1, p. 668. So $\varepsilon(\mathfrak{S})$ is an equivalence system. For coherence, consider any $w, v, u \in W$ such that $v \varepsilon(\mathfrak{S})_{w} u$. Then there is an automorphism $\langle f, g\rangle \in \operatorname{fix}(\mathfrak{S}, w)$ such that $f(v)=u$. $f \subseteq \varepsilon(\mathfrak{S})_{w}$ by construction of $\varepsilon(\mathfrak{S})$, and $f(w)=w$ by construction of fix $(\mathfrak{S}, w)$. To show that $f \in \operatorname{aut}(\varepsilon(\mathfrak{S}))$, consider any $x, y, z \in W$ such that $y \varepsilon(\mathfrak{S})_{x} z$. Then there is a $\xi \in \operatorname{fix}(\mathfrak{S}, x)$ such that $\xi . y=z$. With Part 1 , Lemma 1 (ii), $\langle f, g\rangle . \xi \in$ fix $(\mathfrak{S}, f(x))$. Further, $(\langle f, g\rangle . \xi) . f(y)=\langle f, g\rangle \xi\left\langle f^{-1}, g^{-1}\right\rangle . f(y)=\langle f, g\rangle \xi . y=\langle f, g\rangle . z=$ $f(z)$. Thus $f(y) \varepsilon(\mathfrak{S})_{f(x)} f(z)$. The converse follows by a symmetric argument for $f^{-1}$.

Conversely, it will now be shown that every coherent equivalence system is determined by some structure. Indeed, it will be shown that the relevant structures can be required to be $\times$ closed, where $\times$ may be any sign, i.e., either - or + . This result will then be extended to the analogous claim for Finely generated structures, defined in Part 1, Definition 18. Finally, internally -closed structures and internally + closed structures will be considered, proving that the analogous claim does not hold in the former case, and leaving the issue for the latter case open.

Proposition 3.3. For any sign $\times$, every coherent equivalence system is determined by a $\times$ closed structure.

To keep it readable, the proof will be split into several lemmas. Let $\times$ be a sign and $\approx$ a coherent equivalence system on a set $W$. Let $\mathfrak{F}$ be the frame $\langle W, \emptyset\rangle$. For any $w, v \in W$, define $P_{w}^{v}=\left([v]_{\approx_{w}}\right)_{\mathfrak{F}}$ (recall Part 1, Definition 22); this can be thought of as the propositional intension corresponding to the equivalence class of $v$ under the indistinguishability relation at $w$. The basic idea of the proof is that $\approx$ can be recovered from these equivalence classes, so that one can use the structure $\times$ generated from the structure which contains only the corresponding propositional intensions in its higher-order domains. So define first $\mathfrak{S}=\langle W, \emptyset, B\rangle$ to be the structure such that $B_{W}^{t}=\emptyset$ for all types $t \neq\langle\rangle$ and $B_{w}^{\langle\rangle}=\left\{P_{w}^{v}: v \in W\right\}$ for all $w \in W$. Let $\otimes \mathfrak{S}=\langle W, \emptyset, D\rangle$. It will be shown that $\approx=\varepsilon(\otimes \mathfrak{S})$. As in Part 1 , let $f^{\emptyset}$ be $\langle f, \emptyset\rangle$ for all $f \in S_{W}$.

Lemma 3.4. Let $w, v \in W$ and $f \in S_{W}$ such that $f \subseteq \approx_{w}$. Then $f^{\emptyset} \cdot P_{w}^{v}=P_{w}^{v}$.

Proof. Let $u \in W .\left(f^{\emptyset} \cdot P_{w}^{v}\right)(f(u))=f^{\emptyset} \cdot\left(P_{w}^{v}(u)\right)=f^{\emptyset}\left(\left\{\langle\rangle: u \in[v]_{\approx_{w}}\right\}\right)=\{\langle\rangle$ : $\left.u \in[v]_{\approx_{w}}\right\}=\left\{\langle\rangle: f(u) \in[v]_{\approx_{w}}\right\}=P_{w}^{v}(f(u))$. So $f^{\emptyset} . P_{w}^{v}=P_{w}^{v}$.

Lemma 3.5. Let $f$ be an automorphism of $\approx$. Then for any $w, v \in W, f^{\emptyset} \cdot P_{w}^{v}=$ $P_{f(w)}^{f(v)}$.

Proof. Consider any $u \in W \cdot\left(f^{\emptyset} \cdot P_{w}^{v}\right)(f(u))=f^{\emptyset} \cdot\left(P_{w}^{v}(u)\right)=f^{\emptyset} \cdot\{\langle\rangle: u \in$ $\left.[v]_{\approx_{w}}\right\}=\left\{\langle\rangle: v \approx_{w} u\right\}=\left\{\langle\rangle: f(v) \approx_{f(w)} f(u)\right\}=\left\{\langle\rangle: f(u) \in[f(v)]_{\approx_{f(w)}}\right\}=$ $P_{f(w)}^{f(v)}(f(u))$.

Lemma 3.6. $B \sqsubseteq D$.

Proof. Consider any $w \in W$ and $p \in B_{w}^{T}$. Then $p \in B_{w}^{\langle\rangle}$, so $D \otimes p$. Also, for any $\xi \in \operatorname{fix}(\mathfrak{S}, w), \xi \cdot p=p$, so $p \in D_{w}^{\langle\rangle}$. 
Lemma 3.7. For any $w, v, u \in W, v \approx_{w} u$ iff there is an $f \in S_{W}$ such that $f^{\emptyset} \in \operatorname{fix}(\otimes \mathfrak{S}, w)$ and $f(v)=u$.

Proof. Assume first that $v \approx_{w} u$. By coherence, there is an $f \in \operatorname{aut}(\approx)_{w}$ such that $f(v)=u$ and $f \subseteq \approx_{w}$. By Lemma 3 (ii) of Part 1 , fix $(\mathfrak{S}, w) \subseteq \operatorname{fix}(\otimes \mathfrak{S}, w)$, so it suffices to show that $f^{\emptyset} \in \operatorname{fix}(\mathfrak{S}, w)$. To show that $f^{\emptyset} \in \operatorname{aut}(\mathfrak{S})$, it has to be shown that for all $x \in W$ and types $t, f^{\emptyset} \cdot B_{x}^{t}=B_{f(x)}^{t}$. The claim is trivial for all types $t \neq\langle\rangle . f^{\emptyset} \cdot B_{x}^{\langle\rangle}=f^{\emptyset} \cdot\left\{P_{x}^{y}: y \in W\right\}=\left\{f^{\emptyset} \cdot P_{x}^{y}: y \in W\right\}$. By Lemma 3.5, this is the set $\left\{P_{f(x)}^{f(y)}: y \in W\right\}=\left\{P_{f(x)}^{y}: y \in W\right\}=B_{f(x)}^{\langle\rangle}$. Since $f \in \operatorname{aut}(\approx)_{w}$, $f(w)=w$. Consider any $p \in B_{w}^{T}$. Then $p=P_{w}^{x}$ for some $x \in W$. By Lemma 3.4, $f^{\emptyset} . p=p$. So $f^{\emptyset} \in \operatorname{fix}(\mathfrak{S}, w)$, as required.

Assume now that there is an $f \in S_{W}$ such that $f^{\emptyset} \in \operatorname{fix}(\otimes \mathfrak{S}, w)$ and $f(v)=u$. By Lemma 3.6, $P_{w}^{v} \in D_{w}^{\langle\rangle}$, so $f^{\emptyset} \cdot P_{w}^{v}=P_{w}^{v}$. First, $\left(f^{\emptyset} \cdot P_{w}^{v}\right)(u)=$ $\left(f^{\emptyset} \cdot P_{w}^{v}\right)\left(f^{\emptyset} \cdot v\right)=f^{\emptyset} \cdot\left(P_{w}^{v}(v)\right)=f^{\emptyset} \cdot\left\{\langle\rangle: v \in[v]_{\approx_{w}}\right\}=\{\langle\rangle\}$. Second, $P_{w}^{v}(u)=$ $\left\{\langle\rangle: u \in[v]_{\approx_{w}}\right\}$. So $\{\langle\rangle\}=\left\{\langle\rangle: u \in[v]_{\approx_{w}}\right\}$, and therefore $u \in[v]_{\approx_{w}}$, whence $v \approx_{w} u$.

Proof of Proposition 3.3. Let $w, v, u \in W . v \varepsilon(\otimes \mathfrak{S})_{w} u$ iff there is a $\langle f, g\rangle \in$ fix $(\otimes \mathfrak{S}, w)$ such that $f(v)=u$, which in turn is the case iff there is an $f \in S_{W}$ such that $f^{\emptyset} \in \operatorname{fix}(\otimes \mathfrak{S}, w)$ and $f(v)=u$. By Lemma 3.7, this is the case iff $v \approx_{w} u$. So $\approx=\varepsilon(\otimes \mathfrak{S})$.

Thus, the patterns of local indistinguishability among worlds - which pairs of worlds are indistinguishable at a given world - admitted by all structures, by -closed structures, and by +closed structures all exactly correspond to coherent equivalence systems.

This result can be extended to the class of structures satisfying the constraints on generation imposed in Fine (1977); see Part 1, section 4.4:

Proposition 3.8. Every coherent equivalence system is determined by a Finely generated structure.

Proof. Consider any a coherent equivalence system $\approx$ on a set $W$. Let $\mathfrak{S}=$ $\langle W, I, B\rangle$, where $I=\left\{[v]_{\approx_{w}}: w, v \in W\right\}, B_{W}^{t}=\emptyset$ for all types $t \notin\{e,\langle e\rangle\}$, and for all $w \in W, B_{w}^{e}=\left\{[v]_{\approx_{w}}: v \in W\right\}$ and $B_{w}^{\langle e\rangle}=\{E\}$, where $E(v)=\{p \in I$ : $v \in p\}$ for all $v \in W . \oplus \mathfrak{S}$ is Finely generated, and analogous to the proof of Proposition 3.3 , it can be shown that it determines $\approx$.

The final result of this section shows that the previous results does not extend to the case of internally -closed structures:

Proposition 3.9. Some coherent equivalence system is not determined by any internally - closed structure.

Proof. Let $\approx$ be the equivalence system on $W=\{1,2,3\}$ such that for all $w, v, u \in W, v \approx_{w} u$ iff $(v=w$ iff $u=w)$. It is easy to see that $\approx$ is coherent. Assume for contradiction that there is an internally -closed structure $\mathfrak{S}=$ $\langle W, I, D\rangle$ such that $\varepsilon(\mathfrak{S})=\approx$. Since $2 \approx_{1} 3,2_{\langle W, I\rangle}^{\langle\rangle} \notin D_{1}^{\langle\rangle}$, so there must be a $\left.\xi \in \operatorname{fix}(\mathfrak{S}, w)\right|_{c} \mathfrak{S}$ such that $\xi .1=1$ and $\xi .2 \neq 2$, and therefore $\xi .2=3$. So there must be a cumulative representation of $(23)$ in $D_{W}^{\langle\langle\rangle,\langle\rangle\rangle}$, and hence by negativity of $\mathfrak{S}$ a $w \in W$ such that $\left\{2_{\langle W, I\rangle}^{\langle\rangle}, 3_{\langle W, I\rangle}^{\langle\rangle}\right\} \subseteq D_{w}^{\langle\rangle}$. . 
The analogous question concerning internally +closed structures seems more difficult, and is left open.

\section{Global Indistinguishability}

For any structure $\mathfrak{S}$ and world $w, \operatorname{fix}(\mathfrak{S}, w)$ is the set of automorphisms corresponding to the ways in which modal space can be permuted indistinguishablebly at $w$ in $\mathfrak{S}$. Since these automorphisms are pairs of permutations of worlds and permutations of individuals, it is straightforward to derive a corresponding permutation system: we only have to forget about permutations of individuals.

Definition 4.1. For any structure $\mathfrak{S}=\langle W, I, D\rangle$, the permutation system determined by $\mathfrak{S}$, written $\pi(\mathfrak{S})$, is the function $\pi(\mathfrak{S})$ which maps every $w \in W$ to

$$
\pi(\mathfrak{S})_{w}=\{f: \text { for some } g,\langle f, g\rangle \in \operatorname{fix}(\mathfrak{S}, w)\} .
$$

As in the case of equivalence systems, every permutation system determined by a structure is coherent:

Proposition 4.2. Every structure determines a coherent permutation system.

Proof. Let $\mathfrak{S}=\langle W, I, D\rangle$ be a structure. That $\pi(\mathfrak{S})$ is a permutation system in which each member of $\pi(\mathfrak{S})_{w}$ maps $w$ to itself follows from the fact that for all $w \in W, \operatorname{fix}(\mathfrak{S}, w)$ is a subgroup of $\operatorname{aut}(\mathfrak{S})_{w}$, as noted in Part 1, p. 668. To show that all such permutations are automorphisms of $\pi(\mathfrak{S})$, it suffices to consider any $w, v \in W, f \in \pi(\mathfrak{S})_{w}$ and $g \in \pi(\mathfrak{S})_{v}$, and prove that $f . g \in \pi(\mathfrak{S})_{f(v)}$. Since $f \in \pi(\mathfrak{S})_{w}$ and $g \in \pi(\mathfrak{S})_{v}$, there are $f^{\prime}, g^{\prime} \in S_{I}$ such that $\left\langle f, f^{\prime}\right\rangle \in \operatorname{fix}(\mathfrak{S}, w)$ and $\left\langle g, g^{\prime}\right\rangle \in \operatorname{fix}(\mathfrak{S}, v)$. By Lemma 1 (ii) of Part $1,\left\langle f, f^{\prime}\right\rangle \cdot\left\langle g, g^{\prime}\right\rangle \in \operatorname{fix}(\mathfrak{S}, f(v))$. $\left\langle f, f^{\prime}\right\rangle \cdot\left\langle g, g^{\prime}\right\rangle=\left\langle f . g, f^{\prime} . g^{\prime}\right\rangle$, so $f . g \in \pi(\mathfrak{S})_{f(v)}$.

As in the case of equivalence systems, a converse can be established, showing that every coherent permutation system is determined by some structure. A few tools are needed. First, a strict total order of a set $W$ is a binary relation < which is irreflexive (not $w<w$ ), transitive (if $w<v$ and $v<u$ then $w<u$ ) and total (if $w \neq v$ then $w<v$ or $v<w$ ). Such a relation is a strict wellordering if it is also well-founded (every non-empty set $W^{\prime} \subseteq W$ has a least element, i.e., there is a $w \in W^{\prime}$ such that for all $\left.v \in W^{\prime}, v \nless w\right)$. By the conventions on extending functions to sets and sequences adopted in Part 1, a permutation $f$ of $W$ can be applied to a strict well-ordering $<$ of $W$ : for all $w, v \in W, f(w) f(<) f(v)$ if and only if $w<v$. Similarly, for any set $\Theta$ of strict well-orderings, $f(\Theta)=\{f(<):<\in \Theta\}$. For any permutation group $G$ on $W$ and strict well-ordering $<$ of $W$, let $G(<)$ be $\{f(<): f \in G\}$; this is called the orbit of $<$. If $f$ is a permutation of $W$, then $f G$ is defined as $\{f g: g \in G\}$ and called a left coset of $G$.

The proof strategy is to construct, for a given coherent permutation system $F$, a structure which determines it. On the one hand, this requires including enough in the domain of the structure at a given world $w$ to rule out all automorphisms whose world-permutation is not in $F_{w}$. To do so, the individual domain will be constructed to be empty and the higher-order domains will be constructed to include, for some strict well-ordering $<$ of the worlds, the representation of the property of being a well-ordering to which $<$ is mapped by some 
member of $F_{w}$ - this representation is mapped to itself by an automorphism if and only if its world-permutation is in $F_{w}$. (From a group-theoretic perspective, this follows from the fact that by letting the permutations of a set $W$ act in the natural way on orders on $W$, for every strict well-ordering $<$ of $W$, every permutation group on $W$ becomes the set-wise stabilizer of its own orbit of <.) On the other hand, it has to be ensured that the condition of being an automorphism of the overall structure does not rule out too many permutations for the structure to determine $F$. It turns out that this can be done by including in the domain of a given world $w$ the representations of properties of being a well-ordering to which < is mapped by some member of $F_{w}$ for all strict well-orderings of $W$.

To implement this strategy, the following defines representations of properties of relations among worlds. As for similar cases in Part 1, the upper type index in representations will be dropped where it is clear from context; see, e.g., Definition 24.

Definition 4.3. Let $\mathfrak{F}=\langle W, I\rangle$ be a frame. For any set $\Theta$ of binary relations on $W$, define $\Theta_{\mathfrak{F}}^{\langle\langle\langle\rangle,\langle\rangle\rangle\rangle} \in \iota_{\mathfrak{F}}^{\langle\langle\langle\rangle,\langle\rangle\rangle\rangle}$such that for all $w \in W$ :

$$
\Theta_{\mathfrak{F}}^{\langle\langle\langle\rangle,\langle\rangle\rangle\rangle}(w)=\left\{R_{\mathfrak{F}}^{\langle\langle\rangle,\langle\rangle\rangle}: R \in \Theta\right\}
$$

Lemma 4.4. Let $\mathfrak{F}=\langle W, I\rangle$ be a frame, $\langle f, g\rangle \in \operatorname{aut}(\mathfrak{F})$, and $\Theta$ a set of strict well-orderings of $W$. Then $\langle f, g\rangle . \Theta_{\mathfrak{F}}=(f(\Theta))_{\mathfrak{F}}$.

Proof. Routine, using Lemma 5 (iv) of Part 1.

Theorem 4.5. Every coherent permutation system is determined by a structure.

Proof. Consider any coherent permutation system $F$ on a set $W$. To specify the domain of the structure $\mathfrak{S}$ which will be shown to determine $F$, let $[<]_{G}=$ $\{f(<): f \in G\}_{\langle W, \emptyset\rangle}^{\langle\langle\langle\rangle,\langle\rangle\rangle\rangle}$for any permutation group $G$ on $W$ and strict wellordering $<$ of $W$. Let $\mathfrak{S}$ be the structure $\langle W, \emptyset, D\rangle$ such that for all types $t \neq$ $\langle\langle\langle\rangle,\langle\rangle\rangle\rangle, D_{W}^{t}=\emptyset$, and for all $w \in W$,

$$
D_{w}^{\langle\langle\langle\rangle,\langle\rangle\rangle\rangle}=\left\{[<]_{F_{w}}:<\text { is a strict well-ordering of } W\right\} .
$$

It remains to prove that $F=\pi(\mathfrak{S})$. Consider any $f \in S_{W}$ and $w \in W$. To prove that $f \in F_{w}$ iff $f \in \pi(\mathfrak{S})_{w}$, note the following consequence of Lemma 4.4: For any strict well-ordering $<$ of $W, f^{\emptyset} \cdot[<]_{F_{w}}=[<]_{f F_{w}}$. Call this observation $(*)$.

Assume first that $f \in F_{w}$. To show that $f \in \pi(\mathfrak{S})_{w}$, it has to be shown that $f^{\emptyset} \in \operatorname{fix}(\mathfrak{S}, w)$, which is the case if (i) $f^{\emptyset} \in \operatorname{aut}(\mathfrak{S})$, (ii) $f(w)=w$, and (iii) $f^{\emptyset} \cdot[<]_{F_{w}}=[<]_{F_{w}}$ for any strict well-ordering $<$ of $W$. For (i), consider any $v \in W$; it will be shown that $f^{\emptyset} \cdot D_{v}^{\langle\langle\langle\rangle,\langle\rangle\rangle\rangle}=D_{f(v)}^{\langle\langle\langle\rangle,\langle\rangle\rangle\rangle}$. For $\subseteq$, consider any strict well-ordering $<$ of $W$. By $(*), f^{\emptyset} \cdot[<]_{F_{v}}=[<]_{f F_{v}}=\left\{f g(<): g \in F_{v}\right\}_{\langle W, \emptyset\rangle}=$ $\left\{f . g(f(<)): g \in F_{v}\right\}_{\langle W, \emptyset\rangle}=\left\{g f(<): g \in F_{f(v)}\right\}_{\langle W, \emptyset\rangle}=[f(<)]_{F_{f(v)}} \in D_{f(v)}^{\langle\langle\langle\rangle,\langle\rangle\rangle\rangle}$ (for the penultimate identity, note that $f$ is an automorphism of $F$ ). $\supseteq$ can be derived using inverses. (ii) follows from the coherence of $F$. For (iii), consider any strict well-ordering $<$ of $W$. As observed in $(*), f^{\emptyset} \cdot[<]_{F_{w}}=[<]_{f F_{w}}=[<]_{F_{w}}$ (for the last identity, note that since $f \in F_{w}, f F_{w}=F_{w}$ ).

Assume now that $f \in \pi(\mathfrak{S})_{w}$. Then $f^{\emptyset} \in \operatorname{fix}(\mathfrak{S}, w)$. Let $<$ be any strict well-ordering of $W$. Then $f^{\emptyset} \cdot[<]_{F_{w}}=[<]_{F_{w}}$, so by $(*),[<]_{f F_{w}}=[<]_{F_{w}}$, and therefore $\left\{f g(<): g \in F_{w}\right\}_{\langle W, \emptyset\rangle}=\left\{g(<): g \in F_{w}\right\}_{\langle W, \emptyset\rangle}$. Since the mapping of 
sets of strict well-orderings of $W$ to their representations is injective, $\{f g(<)$ : $\left.g \in F_{w}\right\}=\left\{g(<): g \in F_{w}\right\}$. So there is a $g \in F_{w}$ such that $f(<)=g(<)$. As $<$ is a strict well-ordering of $W, f=g$, and therefore $f \in F_{w}$.

Once this result is in place, it is natural to go on asking whether it extends to $-/+$ closed structures:

Question 4.6. Is every coherent permutation system determined by $a-/+$ closed structure?

This is not obvious, and the question will be left open here. A positive answer could easily be derived from the previous theorem with the claim that symmetries of modal space don't change under generation; i.e., the claim that for all signs $\times$, structures $\mathfrak{S}$ and worlds $w$ : fix $(\mathfrak{S}, w)=\operatorname{fix}(\otimes \mathfrak{S}, w)$. In Part 1 , Lemma 3 (ii), it is shown that $\operatorname{fix}(\mathfrak{S}, w) \subseteq \operatorname{fix}(\otimes \mathfrak{S}, w)$, but it is unclear whether these sets must in fact always be identical. To see how this may fail, note that while $\mathfrak{S}$ need not be a $-/+$ structure, $-/+$ generating imposes the being constraint or its positive weakening; therefore, the domain of $\otimes \mathfrak{S}$ need not include the domain of $\mathfrak{S}$, and consequently, there might be an automorphism in $\operatorname{fix}(\otimes \mathfrak{S}, w)$ not contained in fix $(\mathfrak{S}, w)$.

This observation shows that even if not all coherent permutation systems are determined by a $-/+$ closed structure, then this is in a sense a relatively fragile result. This is because the observation in the last paragraph shows that the match established in Theorem 4.5 can be extended to closed structures in a setting in which neither the being constraint nor its positive weakening are imposed. As noted in Part 1, p. 659, fn. 17, this option is well worth exploring, but beyond the scope of the present investigation; see also Fritz and Goodman (forthcoming). Similarly, the match could be established for +closed structures in a type hierarchy including types of relations of arbitrary ordinal arity. In such a setting, the above proof could be adapted by representing a property of orders of worlds using the corresponding $|W|$-ary relation among world-propositions - the important difference is that such relations trivially satisfy the positive weakening of the being constraint in closed structures.

While Question 4.6 seems difficult to answer, the further strengthening of the hypothesis to internally + closed structures, defined in Part 1, Definition 26, can easily be refuted:

Theorem 4.7. Not every coherent permutation system $F$ is determined by an internally + closed structure.

Proof. Let $W=\{1,2,3,4\}$ and $F$ the permutation system on $W$ such that for all $w \in W, F_{w}=\left(S_{W}\right)_{w}$. It is easy to see that $F$ is coherent. If $\mathfrak{S}$ is a structure such that $F=\pi(\mathfrak{S})$, then for all $w \in W$ and $f \in\left(S_{W}\right)_{w}$, there is a $g$ such that $\langle f, g\rangle \in \operatorname{fix}(\mathfrak{S}, w)$. So in the sense of Definition 33 of Part 1 , for all $w \in W$, the members of $W \backslash\{w\}$ are collectively indistinguishable from $w$, and so by Proposition 21 of Part $1, \mathfrak{S}$ is not internally +closed.

It seems very likely that an analogous result could be given for the class of internally -closed structures, defined in Part 1, Definition 30, but this is omitted here as such structures are found to be implausibly restrictive in Part 1, Section 6.4 


\section{Conclusion}

The equivalence systems and permutation systems determined by structures have all been shown to be coherent. Conversely, the more interesting results established here answer questions of the following form:

Is every coherent ... system determined by a ... structure?

The answers obtained here are summed up in the following table:

\begin{tabular}{l|cc} 
& equivalence & permutation \\
\hline & $\checkmark$ & $\checkmark$ \\
-closed & $\checkmark$ & $?$ \\
+closed & $\checkmark$ & $?$ \\
Finely generated & $\checkmark$ & $?$ \\
internally - closed & $\times$ & $? \times$ \\
internally +closed & $?$ & $\times$
\end{tabular}

Here, the first gap of the above question schema is filled in by a horizontal item, and the second gap by a vertical item; $\checkmark$ indicates Yes, $\times$ No, ? an open question, and ? ${ }^{\times}$a conjectured No.

The results on equivalence systems show that the constraints on patterns of local indistinguishability imposed by all structures, -closed structures, +closed structures, and Finely generated structures are the same, and correspond exactly to the class of coherent equivalence systems; furthermore, it is shown that these results cannot be extended to the class of internally -closed structures. One consequence of this is that -closed structures, +closed structures, and Finely generated structures all admit the same propositional domain functions. (Recall that for any closed structure, a proposition is in the domain of a world $w$ just in case it is true in either both or neither of two worlds indistinguishable at $w$.) Thus no differences between these classes of structures appear on the level of propositional contingency. Furthermore, since equivalence systems uniquely correspond to propositional domain functions, as noted in section 2 , the class of coherent equivalence systems provides a simple and useful way of characterizing the propositional domain functions admitted by these three classes of structures.

As Stalnaker (2012) demonstrates, many of the philosophical issues of higherorder contingency can be illustrated using examples of propositional contingency. Since equivalence systems are vastly simpler than the structures of Part 1 , they will often be preferable as models of propositional contingency. To make the transition from structures to equivalence systems, those who work with a particular class of structures need a characterization of the corresponding class of equivalence systems; this is what the above results provide for the classes of -closed structures, +closed structures, and Finely generated structures.

A concrete application demonstrating the usefulness of equivalence systems concerns the logic of propositional quantifiers: It is easy to show that the set of sentences of higher-order modal logic valid on any of the classes of structures just considered is not recursively axiomatizable. One may therefore wonder whether a highly restrictive fragment, such as the fragment of sentences containing only variables and constants of type \langle\rangle is recursively axiomatizable. This fragment is straightforward to interpret on equivalence systems, and Fritz (forthcoming) shows that the set of sentences in this fragment which are valid on all coherent equivalence systems is recursively isomorphic to full second-order logic, and so 
not recursively axiomatizable. With Propositions 3.2 and 3.3, it follows immediately that the classes of -closed structures, +closed structures, and Finely generated structures all agree with the class of coherent equivalence systems concerning the validities in the propositional fragment of higher-order modal logic, and so that their validities in this fragment are not recursively axiomatizable.

The results on permutation systems obtained here show that the patterns of global indistinguishability admitted by all structures correspond exactly to the coherent permutation systems; furthermore, it is shown that this result cannot be extended to the class of internally +closed structures. As in the case of equivalence systems, this shows that if we are only concerned with patterns of global indistinguishability of arbitrary structures, we may work as well with the simpler coherent permutation systems. Of course, the class of all structures will usually be considered to be too wide. It would therefore be interesting to settle Question 4.6, and in general, provide simple characterizations of the classes of permutation systems determined by structures in one of the various classes of structures considered here.

As noted in section 2, coherent permutation systems draw finer distinctions than coherent equivalence systems. Given the results in the last two sections, this can be used to conclude that in the class of all structures, the patterns of global indistinguishability outrun the patterns of local indistinguishability: some structures share the same pattern of local indistinguishability while displaying different patterns of global indistinguishability. Since it has not been established which permutation systems are determined by any of the other classes of structures, this insight cannot straightforwardly be extended to these classes. Especially for the highly restrictive class of internally +closed structures, it seems possible that the difference between the patterns of global and local indistinguishability disappears. The following result shows that this is not the case. Since all internally + closed structures are +closed (see Part 1, Proposition 13) this result extends to + closed structures.

Proposition 5.1. There are internally + closed structures which determine the same equivalence systems but different permutation systems.

Proof. Define $\mathfrak{S}=\langle W, I, B\rangle$ and $\mathfrak{S}^{\prime}=\left\langle W, I, B^{\prime}\right\rangle$ such that $W=\{1,2,3,4,5\}$, $I=\{2,3,4\}, B_{W}^{t}=B_{W}^{\prime t}=\emptyset$ for all types $t \notin\{e,\langle e, e\rangle\}$ and

$$
\begin{aligned}
& B_{1}^{e}=B_{1}^{\prime e}=\emptyset \\
& B_{i}^{e}=B_{i}^{\prime e}=I \backslash\{i\} \text { for all } i \in\{2,3,4\} \\
& B_{5}^{e}=B_{5}^{\prime e}=I \\
& B_{i}^{\langle e, e\rangle}=\emptyset \text { and } B_{i}^{\prime\langle e, e\rangle}=\{<\} \text { for all } i \in W
\end{aligned}
$$

where $\left\langle\in \iota_{\langle W, I\rangle}^{\langle e, e\rangle}\right.$ such that for all $i \in W$,

$$
<(i)=\{\langle 2,3\rangle,\langle 3,4\rangle,\langle 4,2\rangle\} \cap\left(B_{i}^{\prime e}\right)^{2}
$$

It will be shown that $\oplus \mathfrak{S}$ and $\oplus \mathfrak{S}^{\prime}$ witness the claim to be established. Since the representations of all permutations of both $W$ and $I$ are in the relevant domain of 5 in both $\oplus \mathfrak{S}$ and $\oplus \mathfrak{S}^{\prime}$, they are internally +closed. To see that $\pi(\oplus \mathfrak{S}) \neq \pi\left(\oplus \mathfrak{S}^{\prime}\right)$, note that $(23) \in \pi(\oplus \mathfrak{S})_{1}$ but $(23) \notin \pi\left(\mathfrak{S}^{\prime}\right)_{1}$. To see that 
$\varepsilon(\oplus \mathfrak{S})=\varepsilon\left(\oplus \mathfrak{S}^{\prime}\right)$, note that both of them are identical to the equivalence system $\approx$ such that $\approx_{i}=\mathrm{id}_{W}$ for all $i \in\{2,3,4,5\}$, and $\approx_{1}$ is the equivalence relation corresponding to the partition $\{\{1\},\{2,3,4\},\{5\}\}$.

\section{References}

Kit Fine. Properties, propositions and sets. Journal of Philosophical Logic, 6 (1):135-191, 1977.

Peter Fritz. Propositional contingentism. The Review of Symbolic Logic, 9(1): 123-142, 2016.

Peter Fritz. Logics for propositional contingentism. The Review of Symbolic Logic, forthcoming.

Peter Fritz and Jeremy Goodman. Higher-order contingentism, part 1: Closure and generation. Journal of Philosophical Logic, 45(6):645-695, 2016.

Peter Fritz and Jeremy Goodman. Counting incompossibles. Mind, forthcoming.

Robert Stalnaker. Mere Possibilities. Princeton: Princeton University Press, 2012.

Robert Stalnaker. Models and reality. Canadian Journal of Philosophy, 46(4-5): 709-726, 2016. 